Chapter 38.

\title{
Strawberry Production in Florida
}

N.A. Peres, J.F. Price, W.M. Stall, C.K. Chandler, S.M. Olson, T.G. Taylor, S.A. Smith, E.H. Simonne

\section{BOTANY}

Family - Rosaceae

Strawberry - Fragaria $x$ ananassa

\section{Origin}

The cultivated strawberry is of recent origin resulting from a cross between $F$. chiloensis and $F$. virginiana. The former is native to the west coast of North and South America and the latter is native to the east coast of North America.

\section{Related Species}

Strawberry is the only vegetable crop in the Rosaceae family. However, a large number of ornamental plants and important tree fruits such as apple, pear, cherry, and plum are included in this family.

\section{VARIETIES}

Strawberry varieties grown in Florida are:

Camarosa

Carmine

Camino Real

Gaviota

Strawberry Festival

Sweet Charlie

Treasure

Ventana

Winter Dawn

\section{POLLINATION}

Pollination of all pistils of the strawberry flower and subsequent fertilization results in maximum fruit size and attainment of shape typical of the variety. Although many insects may effect the transfer of pollen from the anthers to the pistil, honeybees are the most effective transfer agent. Accordingly, populations of wild bees in the vicinity of strawberry fields should be encouraged, care in pesticide application exercised, and possible use of rented domesticated bees considered.
PLANTING

Two types of transplants are used to begin fruiting fields in Florida, bare-root green-top plants and containerized transplants. Bare-root transplants are the most widely available type of transplant but are more difficult to establish in the field. These transplants require overhead sprinkler irrigation during the hottest part of the day for the first 7 to 12 days after planting. This irrigation reduces wilting and leaf loss while the plant develops a root system sufficient to support itself. Containerized transplants require much less overhead irrigation for reestablishment. Regardless of the type of transplant used, it is important not to set the transplant too deep, covering the crown, or too shallow, leaving roots exposed.

\section{FERTILIZER AND LIME}

For sprinkler-irrigated crops, broadcast all $\mathrm{P}_{2} \mathrm{O}_{5}$, micronutrients and $25 \%$ of $\mathrm{N}$ and $\mathrm{K}_{2} \mathrm{O}$ before mulch bedding. Place remaining $\mathrm{N}$ and $\mathrm{K}_{2} \mathrm{O}$ in band in center of bed 3 to 4 inches deep. Slow-release $\mathrm{N}$ can supply a portion of $\mathrm{N}$ requirement (25\%) applied in the broadcast material. Soil test results for strawberry on mineral soils are given in Table 2.

Table 1. Planting information for strawberry.

\begin{tabular}{||lc||}
\hline \multicolumn{2}{|c|}{ Planting dates } \\
North Florida & Sept 20 - Oct.15 \\
Central Florida & Sept 25 - Oct 25 \\
South Florida & Oct 1 - Dec 1 \\
\hline \hline Establishment information & 2-row beds \\
Distance between beds (in.) & 48 - 60 \\
Distance between plants (in.) & $12-16$ \\
Distance between rows (in.) & $12-14$ \\
\hline \hline Days to first ripe fruit & $40-601$ \\
Plant populations ${ }^{2}$ (acre) & $16,000-22,000$ \\
\hline \hline 1 From transplanting date. \\
2 Populations based on closest between and within row spacing. \\
\hline
\end{tabular}


For drip-irrigated crops broadcast all $\mathrm{P}_{2} \mathrm{O}_{5}$, micronutrients and up to 20 to $25 \%$ of $\mathrm{N}$ and $\mathrm{K}_{2} \mathrm{O}$ in before bedding. Apply remaining $\mathrm{N}$ and $\mathrm{K}_{2} \mathrm{O}$ through drip tube with the schedule given in Table 3 , starting 2 to 3 weeks after planting.

\section{PLANT TISSUE ANALYSIS}

Plant tissue analysis information for strawberry is given in Table 4. The analysis is done at first harvest using the most recently matured leaf.

\section{PETIOLE SAP TESTING}

Fresh sap can be pressed from leaf petioles and analyzed for nitrogen and potassium concentrations. Results can be used to make adjustments in the fertilization program. Sufficiency ranges for sap testing for strawberry are presented in Table 5.

\section{IRRIGATION}

Initial water requirements (see Chapter 8, Principles and Practices for Irrigation Management of Vegetables, Table 4 to 6) of strawberry transplants will be low (20\% to $40 \%$ of ETo, see Chapter 8, Principles and Practices for Irrigation

Table 2. Soil test results and fertilizer recommendations for strawberry on 4-foot bed centers on mineral soils. ${ }^{1}$

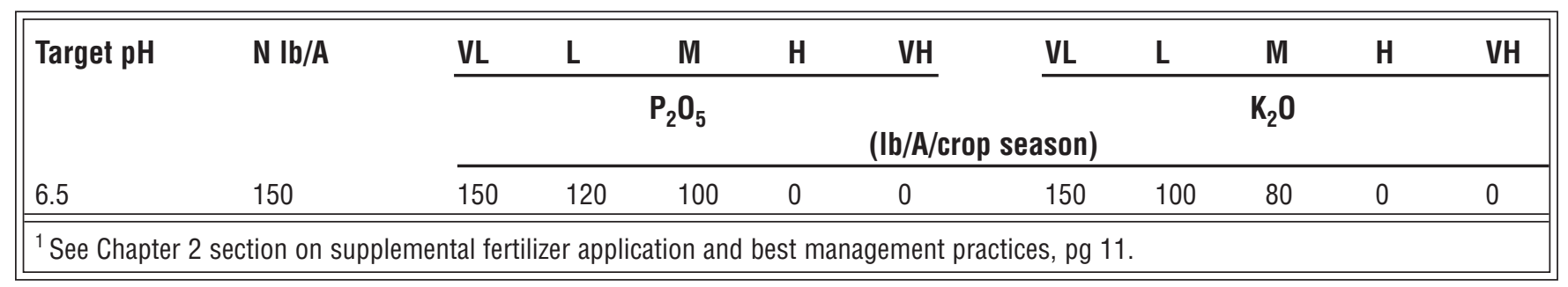

Table 3. Fertilization recommendations for strawberry grown in central Florida on sandy soils testing very low in Mehlich-1 potassium $\left(\mathrm{K}_{2} \mathrm{O}\right)$

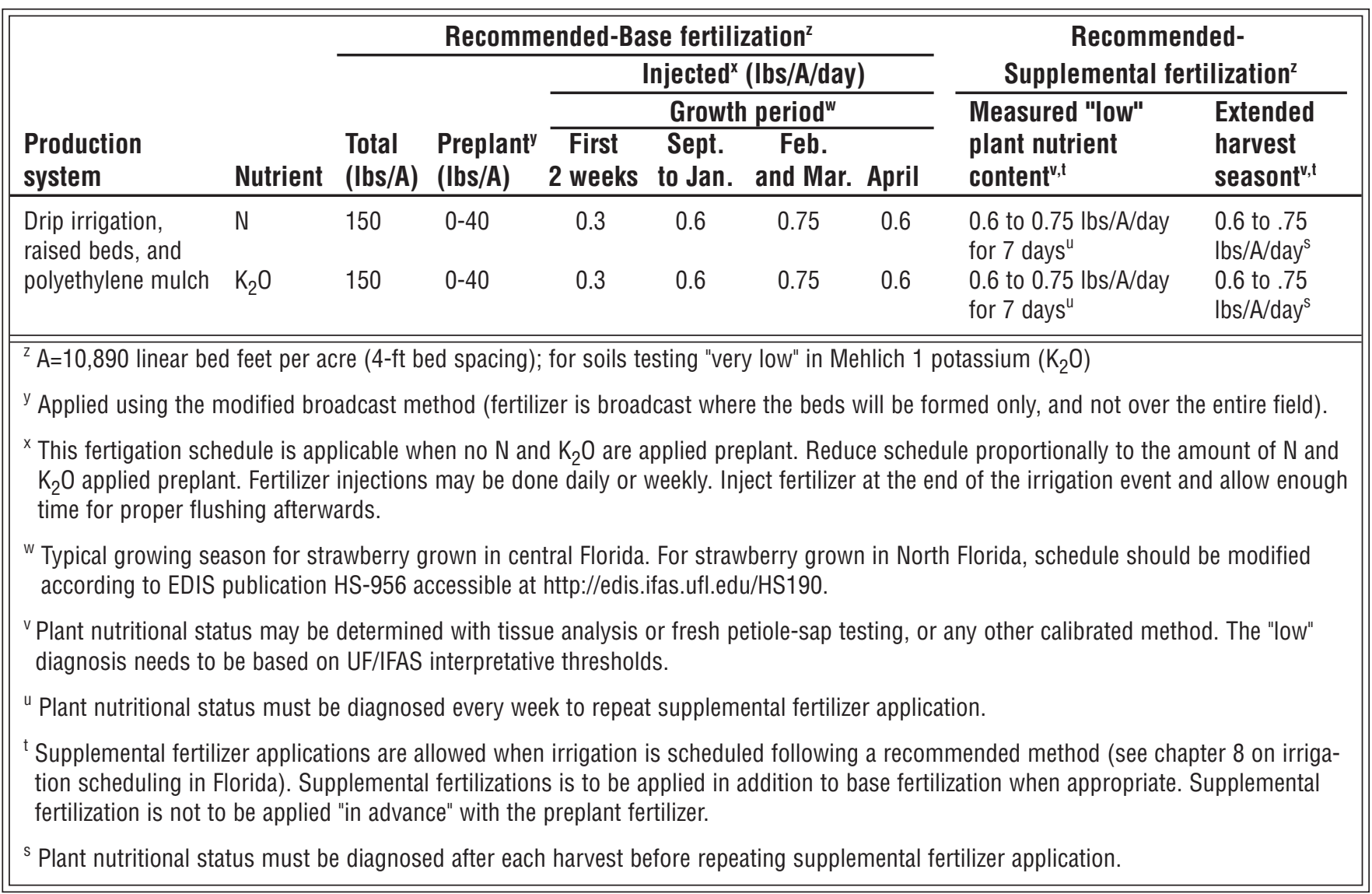


Management of Vegetables, Table 3). As plants grow, midseason water requirements will gradually approach $50 \%$ of ETo for drip irrigated plants and $70 \%$ of ETo for overhead irrigated plants. Water requirements during late season growth will average $60 \%$ of ETo for drip irrigated plants and $85 \%$ of ETo for overhead irrigated plants. Field conditions that are too wet may promote undesirable disease conditions for plants and fruit. Therefore, irrigations should be scheduled to maintain proper bed moisture levels without resulting in excessive moisture or prolonged wetness to plants or row middles.

Sprinkler irrigation also is needed to help establish transplants. Sprinkling is needed during the hot part of the day to keep transplants from wilting and to minimize leaf loss. Irrigation for transplant establishment is usually needed for the first 7 to 12 days of plant establishment.

\section{WEED MANAGEMENT}

Herbicides labeled for weed control in strawbery are listed in Table 6.

\section{DISEASE MANAGEMENT}

Several fungal diseases cause losses each season in Florida. These include Botrytis fruit rot (Botrytis cinerea), anthracnose fruit rot (Colletotrichum acutatum), Colletotrichum crown rot (Colletotrichum gloeosporioides), powdery mildew (Sphaerotheca macularis), and Phytophthora wilt and crown rot (Phytophthora citricola and $P$. cactorum). The only bacterial disease of importance is angular leaf spot (Xanthomonas fragariae).
Control of these diseases typically relies upon the use of disease free transplants and preventative applications of protectant fungicides. We currently recommend that growers apply protectant fungicides weekly throughout the season. Additional fungicide treatments should be made to control specific disease problems when they develop or when experience suggests they may develop. Chemicals approved for disease management on strawberry are listed in Table 7. Peak disease periods for south Florida are shown in Fig. 38-1.

\section{INSECT AND MITE MANAGEMENT}

Table 8 outlines the insecticides adn miticides approved for use on strawberry.

\section{PRODUCTION COSTS}

An example of breakeven production costs for strawberry are given in Table 9 .

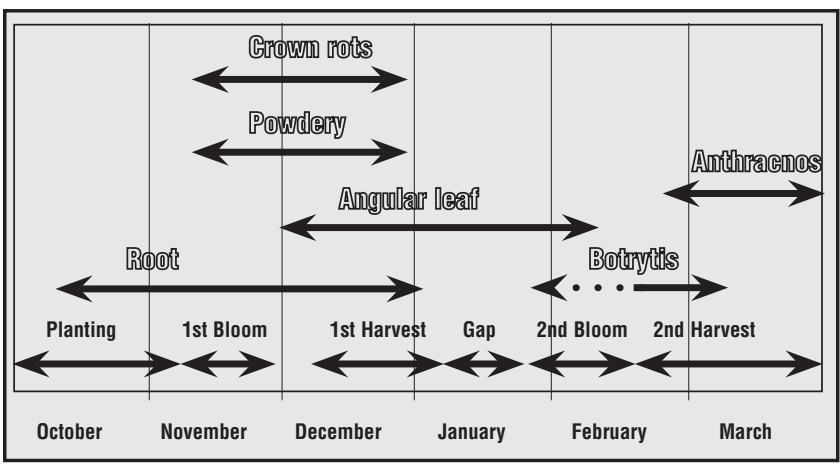

Fig. 38-1. Peak disease periods for south Florida.

Table 4. Plant tissue analysis for strawberry at first harvest. Dry wt. basis.

\begin{tabular}{|c|c|c|c|c|c|c|c|c|c|c|c|c|}
\hline \multirow[b]{2}{*}{ Status } & N & $\mathbf{P}$ & K & $\mathrm{Ca}$ & $\mathrm{Mg}$ & S & $\mathrm{Fe}$ & $\mathrm{Mn}$ & $\mathrm{Zn}$ & B & $\mathrm{Cu}$ & Mo \\
\hline & \multicolumn{6}{|c|}{ Percent } & \multicolumn{6}{|c|}{ Parts per million } \\
\hline Deficient & $<3.0$ & 0.2 & 1.5 & 0.4 & 0.25 & 0.2 & 50 & 30 & 20 & 20 & 5 & 5.0 \\
\hline Adequate range & $3.0-3.5$ & $0.2-0.4$ & $1.5-2.5$ & $0.4-1.5$ & $0.25-0.50$ & $0.2-0.6$ & $50-100$ & $30-100$ & $20-40$ & $20-40$ & $5-10$ & $5.0-8.0$ \\
\hline High & $>3.5$ & 0.4 & 2.5 & 1.5 & 0.50 & 0.6 & 100 & 100 & 40 & 40 & 10 & 8.0 \\
\hline Toxic (>) & & & & & & & & 800 & & & & \\
\hline
\end{tabular}

Table 5. Sufficiency ranges for petiole sap testing for strawberry grown in Central Florida.

\begin{tabular}{|c|c|c|}
\hline \multirow[t]{2}{*}{ Crop development stage } & \multicolumn{2}{|c|}{ Fresh petiole sap concentration (ppm) } \\
\hline & $\mathrm{NO}_{3}-\mathrm{N}$ & $\mathbf{K}$ \\
\hline November, soon after planting & $800-900$ & $3000-3500$ \\
\hline December, first harvesting & $600-800$ & $3000-3500$ \\
\hline January, main season & $600-800$ & $2500-3000$ \\
\hline February, main season & $300-500$ & $2000-2500$ \\
\hline March, main season & $200-500$ & $1800-2500$ \\
\hline April, late harvest, near end of season & $200-500$ & $1500-2000$ \\
\hline
\end{tabular}


Table 6. Chemical weed controls: strawberries.

\begin{tabular}{|c|c|c|c|c|}
\hline \multirow[b]{2}{*}{ Herbicide } & \multirow[b]{2}{*}{ Labeled crops } & \multirow{2}{*}{$\begin{array}{l}\text { Time of } \\
\text { application to crop }\end{array}$} & \multicolumn{2}{|c|}{ Rate (Ibs. Al./Acre) } \\
\hline & & & Mineral & Muck \\
\hline $\begin{array}{l}\text { Carfentrazone } \\
\text { (Aim) }\end{array}$ & Strawberry & $\begin{array}{l}\text { Preplant } \\
\text { Directed-hooded } \\
\text { Row-middles }\end{array}$ & 0.031 & 0.031 \\
\hline
\end{tabular}

Remarks: Aim may be applied as a preplant burndown treatment and/or as a post-directed hooded application to row middles for the burndown of emerged broadleaf weeds. May be tank mixed with other registered herbicides. May be applied at up to $2 \mathrm{lz}$ ( $0.031 \mathrm{lb}$ ai). Use a quality spray adjuvant such as crop oil concentrate (coc) or non-ionic surfactant at recommended rates.

\begin{tabular}{l}
\hline \hline Clethodim Strawberry \\
(Select)
\end{tabular}

\begin{tabular}{|c|c|c|c|}
\hline $\begin{array}{l}\text { Glyphosate } \\
\text { (Roundup, Durango } \\
\text { Touchdown, Glyphomax) }\end{array}$ & Strawberry & $\begin{array}{l}\text { Chemical fallow } \\
\text { Preplant } \\
\text { Pre transplant }\end{array}$ & $0.3-1.0$ \\
\hline
\end{tabular}

Remarks: Roundup, Glyphomax and Touchdown have several formulations. Check the label of each for specific labeling directions.

\begin{tabular}{|c|c|c|c|}
\hline $\begin{array}{l}\text { Napropamide } \\
\text { (Devrinol 12E) }\end{array}$ & Strawberry & $\begin{array}{l}\text { Posttransplant and } \\
\text { established plants }\end{array}$ & $2.0-4.0$ \\
\hline
\end{tabular}

(Devrinol 10G)

Remarks: Controls annual grasses and annual broadleaf weeds. Water in to a depth of 2 to 4 inches. Does not control established weeds. Do not apply from bloom to harvest.

\begin{tabular}{|llll}
\hline \hline $\begin{array}{l}\text { Oxyfluorfen } \\
\text { (Goal } 2 \mathrm{XL})\end{array}$ & Strawberry & Fallow bed prior to transplanting & $0.25-0.5$ \\
(Goaltender) & & Mulch culture & --- \\
\hline
\end{tabular}

Remarks: Must have a 30-day treatment to planting interval. Mulch may be put down any time during this period. Apply as a preemergence broadcast or banded treatment to pre-formed beds as a fallow bed application.

\begin{tabular}{|llll|}
\hline \hline $\begin{array}{l}\text { Paraquat } \\
\text { (Gramoxone Inteon) }\end{array}$ & Strawberry & Postemergence directed spray & 0.47
\end{tabular}

Remarks: Postemergence directed spray. For control of emerged annual broadleaf weeds and grasses and for top kill and suppression of emerged perennial weeds between the rows after crop emergence or establishment. Apply 1.2 pts Gramoxone Extra or 1.6 pts of Boa per sprayed acre in a minimum of 20 gals. spray mix. Use shields to prevent spray contact with crop plants. Use a non-ionic surfactant. Do not apply more than 3 times per crop season. Do not apply within 3 days of harvest.

\begin{tabular}{|lll|}
\hline \hline $\begin{array}{l}\text { Paraquat } \\
\text { (Gramoxone Inteon) }\end{array}$ & Strawberry & $\begin{array}{l}\text { Post Harvest directed spray } \\
\text { Desiccation }\end{array}$ \\
\hline
\end{tabular}

Remarks: Special Local Needs (24c) Label for desiccation of strawberry plants following harvest. Add a non-ionic surfactant or crop oil concentrated to the spray mix.

\begin{tabular}{|c|c|c|c|}
\hline $\begin{array}{l}\text { Pelargonic Acid } \\
\text { (Scythe) }\end{array}$ & Strawberry & $\begin{array}{l}\text { Preplant } \\
\text { Directed-Shielded }\end{array}$ & $3-10 \% \mathrm{v} / \mathrm{v}$ \\
\hline
\end{tabular}

Remarks: Product is a contact, nonselective, foliar herbicide. It has no residual control. May be tank mixed with soil residual herbicides. Consult label for rates.

\begin{tabular}{|c|c|c|c|c|}
\hline $\begin{array}{l}\text { Terbacil } \\
\text { (Sinbar) }\end{array}$ & Strawberry & Pretransplant & 0.2 & --- \\
\hline
\end{tabular}

Remarks: Make a once-per-year application of 4 oz. of Sinbar per acre after bedding but prior to transplanting. Note - a 110 day phi is on label. Check rotational crop limitations. 
Table 7. Fungicides approved for disease management of strawberry diseases in Florida

\begin{tabular}{|c|c|c|c|c|c|c|}
\hline Chemical & $\begin{array}{l}\text { Fungicide } \\
\text { Groupa }\end{array}$ & $\frac{\text { Maximum }}{\text { Application }}$ & $\begin{array}{l}\text { Rate/Acre/ } \\
\text { Season }\end{array}$ & $\begin{array}{l}\text { Min. Days } \\
\text { to Harvest }\end{array}$ & $\begin{array}{l}\text { Pertinent Diseases } \\
\text { or Pathogens }\end{array}$ & Remarks \\
\hline $\begin{array}{l}\text { Abound } \\
\text { (azoxystrobin) }\end{array}$ & 11 & $15.4 \mathrm{fl} \mathrm{ozs}$ & 1.92 qts & 0 & $\begin{array}{l}\text { Anthracnose } \\
\text { Powdery mildew } \\
\text { Botrytis (suppression } \\
\text { only) }\end{array}$ & $\begin{array}{l}\text { Do not make more than } 2 \\
\text { sequential applications and no } \\
\text { more than } 4 \text { applications per } \\
\text { crop year. See label for instruc- } \\
\text { tions on dipping transplants }\end{array}$ \\
\hline $\begin{array}{l}\text { Aliette WDG1 } \\
\text { (fosetyl-Al) }\end{array}$ & 33 & $5 \mathrm{lbs}$ & $30 \mathrm{lbs}$ & 12 hours & Phytophthora diseases & $\begin{array}{l}\text { Do not tank mix with copper } \\
\text { fungicides }\end{array}$ \\
\hline $\begin{array}{l}\text { Cabrio EG } \\
\text { (pyraclostrobin) }\end{array}$ & 11 & $14 \mathrm{fl} \mathrm{ozs}$ & $70 \mathrm{fl} \mathrm{ozs}$ & 0 & $\begin{array}{l}\text { Anthracnose } \\
\text { Leaf spot } \\
\text { Powdery mildew } \\
\text { Botrytis (suppression } \\
\text { only) }\end{array}$ & $\begin{array}{l}\text { Do not make more than } 2 \\
\text { sequential applications and no } \\
\text { more than } 5 \text { applications per } \\
\text { crop year }\end{array}$ \\
\hline $\begin{array}{l}\text { Captan } 50 \text { WP } \\
\text { (captan) }\end{array}$ & M3 & $6 \mathrm{lbs}$ & $48 \mathrm{lbs}$ & 12 & $\begin{array}{l}\text { Anthracnose } \\
\text { Botrytis fruit rot } \\
\text { Leaf spot }\end{array}$ & $\begin{array}{l}\text { Rate per treated acre. Special } \\
\text { label for FL allows up to } 24 \\
\text { applications per season }\end{array}$ \\
\hline $\begin{array}{l}\text { Captan } 80 \text { WDG } \\
\text { (captan) }\end{array}$ & M3 & $3.75 \mathrm{lbs}$ & $30 \mathrm{lbs}$ & 12 & $\begin{array}{l}\text { Anthracnose } \\
\text { Botrytis fruit rot } \\
\text { Leaf spot }\end{array}$ & $\begin{array}{l}\text { Rate per treated acre. Special } \\
\text { label for FL allows up to } 24 \\
\text { applications per season }\end{array}$ \\
\hline $\begin{array}{l}\text { Captec 4L } \\
\text { (captan) }\end{array}$ & M3 & 3 qts & $24 \mathrm{qts}$ & $1^{2}$ & $\begin{array}{l}\text { Anthracnose } \\
\text { Botrytis fruit rot, } \\
\text { Leaf spot }\end{array}$ & $\begin{array}{l}\text { Rate per treated acre. Special } \\
\text { label for FL allows up to } 24 \\
\text { applications per season }\end{array}$ \\
\hline $\begin{array}{l}\text { Captevate } 68 \text { WDG } \\
\text { (captan + fenhexa- } \\
\text { mid) }\end{array}$ & $\mathrm{M} 3+17$ & $5.25 \mathrm{lbs}$. & $21 \mathrm{lbs}$. & 0 & $\begin{array}{l}\text { Botrytis fruit rot } \\
\text { Anthracnose }\end{array}$ & $\begin{array}{l}\text { Do not make more than } 2 \text { con- } \\
\text { secutive applications }\end{array}$ \\
\hline $\begin{array}{l}\text { Copper } \\
\text { (many brands) }^{3}\end{array}$ & M1 or M9 & varies & varies & $1-2$ & Angular leaf spot & $\begin{array}{l}\text { Frequent use of copper fungi- } \\
\text { cides may cause foliar burn }\end{array}$ \\
\hline $\begin{array}{l}\text { Elevate } 50 \text { WDG } \\
\text { (fenhexamid) }\end{array}$ & 17 & $1.5 \mathrm{lbs}$ & $6 \mathrm{lbs}$ & 0 & Botrytis fruit rot & $\begin{array}{l}\text { Do not make more than } 2 \text { con- } \\
\text { secutive applications }\end{array}$ \\
\hline $\begin{array}{l}\text { Nova 40W } \\
\text { (myclobutanil) }\end{array}$ & 3 & $5 \mathrm{oz}$. & $30 \mathrm{oz}$. & 0 & $\begin{array}{l}\text { Powdery mildew, } \\
\text { Leaf spot, } \\
\text { Leaf blight }\end{array}$ & $\begin{array}{l}\text { Do not plant rotational crops } \\
\text { until } 30 \text { days after last applica- } \\
\text { tion }\end{array}$ \\
\hline $\begin{array}{l}\text { Potassium bicarbon- } \\
\text { ate (many brands) }\end{array}$ & & varies & varies & 1 & Powdery mildew & $\begin{array}{l}\text { Do not mix with highly acid } \\
\text { products }\end{array}$ \\
\hline $\begin{array}{l}\text { Potassium phosphate } \\
\text { (many brands) }^{5}\end{array}$ & & varies & varies & 0 & Phytophthora diseases & $\begin{array}{l}\text { May cause foliar burn if applied } \\
\text { with copper based products }\end{array}$ \\
\hline $\begin{array}{l}\text { Pristine } \\
\text { (pyraclostrobin + } \\
\text { boscalid) }\end{array}$ & $11+7$ & 23 ozs & 115 ozs & 0 & $\begin{array}{l}\text { Botrytis fruit rot } \\
\text { Anthracnose } \\
\text { Powdery mildew } \\
\text { Leaf spot }\end{array}$ & $\begin{array}{l}\text { Do not make more than } 2 \text { con- } \\
\text { secutive applications and no } \\
\text { more than } 5 \text { applications per } \\
\text { crop }\end{array}$ \\
\hline $\begin{array}{l}\text { Procure 50WS } \\
\text { (triflumizole) }\end{array}$ & 3 & $80 z$. & $32 \mathrm{oz}$ & 1 & Powdery mildew & $\begin{array}{l}\text { Do not plant leafy vegetables } \\
\text { within } 30 \text { days or root vegeta- } \\
\text { bles within } 60 \text { days or rotational } \\
\text { crops not on label for one year } \\
\text { after application }\end{array}$ \\
\hline $\begin{array}{l}\text { Ridomil Gold EC } \\
\text { (metalaxyl-M) }\end{array}$ & 4 & $\begin{array}{l}1 \mathrm{pt} / \mathrm{trtd} . \\
\text { acre }\end{array}$ & $\begin{array}{l}1 \frac{1}{2} \text { qts/trtd } \\
\text { acre }\end{array}$ & & Phytophthora diseases & $\begin{array}{l}\text { See label for use in drip irriga- } \\
\text { tion }\end{array}$ \\
\hline $\begin{array}{l}\text { Rovral } 46 \\
\text { (iprodione) }\end{array}$ & 2 & $2 \mathrm{pts}$ & $2 \mathrm{pts}$ & $\mathrm{N} / \mathrm{A}$ & $\begin{array}{l}\text { Botrytis fruit rot, } \\
\text { Stem end rot, } \\
\text { Phomopsis soft rot, } \\
\text { Leaf spot, } \\
\text { Rhizoctonia diseases }{ }^{7}\end{array}$ & $\begin{array}{l}\text { Do not make more than } 1 \text { appli- } \\
\text { cation per season. Do not apply } \\
\text { after bloom initiation }\end{array}$ \\
\hline
\end{tabular}


Table 7. Continued.

\begin{tabular}{|c|c|c|c|c|c|c|}
\hline Chemical & $\begin{array}{l}\text { Fungicide } \\
\text { Group }^{\mathrm{a}}\end{array}$ & $\frac{\text { Maximum }}{\text { Application }}$ & $\begin{array}{l}\text { Rate/Acre/ } \\
\text { Season }\end{array}$ & $\begin{array}{l}\text { Min. Days } \\
\text { to Harvest }\end{array}$ & $\begin{array}{l}\text { Pertinent Diseases } \\
\text { or Pathogens }\end{array}$ & Remarks \\
\hline $\begin{array}{l}\text { Rovral } 75 \text { WG } \\
\text { (iprodione) }\end{array}$ & 2 & 1.33 & 1.33 & $\mathrm{~N} / \mathrm{A}$ & $\begin{array}{l}\text { Botrytis fruit rot, } \\
\text { Stem end rot, } \\
\text { Phomopsis soft rot, } \\
\text { Leaf spot, } \\
\text { Rhizoctonia diseases } 7\end{array}$ & $\begin{array}{l}\text { Do not make more than } 1 \text { appli- } \\
\text { cation per season. Do not apply } \\
\text { after bloom initiation }\end{array}$ \\
\hline $\begin{array}{l}\text { Scala SC } \\
\text { (pyrimethanil) }\end{array}$ & 9 & 18 fl. $0 z$. & $54 \mathrm{fl} .0 \mathrm{z}$. & 1 & Botrytis fruit rot & $\begin{array}{l}\text { Do not make more than } 2 \text { con- } \\
\text { secutive applications. Do not } \\
\text { use more than } 2 \text { of } 6 \text { applica- } \\
\text { tions per season }\end{array}$ \\
\hline $\begin{array}{l}\text { Serenade Max } \\
\text { (Bacillus subtilis) }\end{array}$ & & $3 \mathrm{lbs}$. & & 0 & $\begin{array}{l}\text { Powdery mildew, } \\
\text { Botrytis fruit rot, } \\
\text { Anthracnose }\end{array}$ & $\begin{array}{l}\text { Should to be used in combina- } \\
\text { tion with other fungicides }\end{array}$ \\
\hline $\begin{array}{l}\text { Sulfur } \\
\text { (many brands) }^{8}\end{array}$ & M1 or M9 & varies & varies & 1 & Powdery mildew & Do not use during hot weather \\
\hline $\begin{array}{l}\text { Switch } 62.5 \text { WG } \\
\text { (cyprodinil + fludiox- } \\
\text { onil) }\end{array}$ & $9+12$ & 14 ozs & 56 ozs & 0 & $\begin{array}{l}\text { Botrytis fruit rot } \\
\text { Anthracnose }\end{array}$ & $\begin{array}{l}\text { Do not make more than } 2 \text { con- } \\
\text { secutive applications. Do not } \\
\text { plant crops not on the label for } \\
30 \text { days after last application }\end{array}$ \\
\hline $\begin{array}{l}\text { Thiram } 65 \text { WSB } \\
\text { (thiram) }\end{array}$ & M2 & $5 \mathrm{lbs}$ & $25 \mathrm{lbs}$ & 3 & Botrytis fruit rot & $\begin{array}{l}\text { Do not rotate treated crops with } \\
\text { other crops for which Thiram is } \\
\text { not registered }\end{array}$ \\
\hline $\begin{array}{l}\text { Topsin } 4.5 \mathrm{~L} \\
\text { (thyophanate-methyl) }\end{array}$ & 1 & 20 fl. $0 z$. & 80 fl. $0 z$. & 1 & $\begin{array}{l}\text { Botrytis fruit rot, } \\
\text { Colletotrichum crown rot, } \\
\text { Leaf scorch, } \\
\text { Leaf blight, Powdery } \\
\text { mildew }\end{array}$ & $\begin{array}{l}\text { Do not use Topsin alone. } \\
\text { Fungicides from different chem- } \\
\text { ical groups should be used } \\
\text { in spray program for disease } \\
\text { resistance management }\end{array}$ \\
\hline $\begin{array}{l}\text { Topsin M } 70 \text { W, } \\
\text { Topsin M WSB } \\
\text { (thyophanate-methyl) }\end{array}$ & 1 & $1 \mathrm{lb}$ & $4 \mathrm{lbs}$ & 1 & $\begin{array}{l}\text { Botrytis fruit rot, } \\
\text { Colletotrichum crown rot, } \\
\text { Leaf scorch, } \\
\text { Leaf blight, Powdery } \\
\text { mildew }\end{array}$ & $\begin{array}{l}\text { Do not use Topsin alone. } \\
\text { Fungicides from different chem- } \\
\text { ical groups should be used } \\
\text { in spray program for disease } \\
\text { resistance management }\end{array}$ \\
\hline \multicolumn{7}{|c|}{$\begin{array}{l}\text { N/A - Not available } \\
1 \text { See label for instructions for dip and foliar treatments for red stele } \\
2 \text { Unless protective clothing is worn } \\
3 \text { e.g. Kocide, Champion, Champ, Basicop, Cuprofix Disperss, Copper Count-N, Nordox, Nu Cop } \\
4 \text { e.g. Kaligreen, Armicarb, Milstop } \\
5 \text { e.g. Fosphite, Helena Prophyt } \\
6 \text { 2pts./100 gal. water may be used as a preplant dip immediately prior to planting } \\
7 \text { Not listed on label } \\
8 \text { e.g. Micro Sulf, Enduro, Sulfur } 90 W, \text { Super-Six, Microthiol Disperss, Wettable Sulfur, Kumulus } \\
\text { a Fungicide group (FRAC Code): Numbers (1-37) and letters (M, U, P) are used to distinguish the fungicide mode of action groups. All } \\
\text { fungicides within the same group (with same number or letter) indicate same active ingredient or similar mode of action. This informa- } \\
\text { tion must be considered for the fungicide resistance management decisions. M = Multi site inhibitors, fungicide resistance risk is low; } \\
U=\text { Recent molecules with unknown mode of action; P = host plant defense inducers. Source: http://www.frac.info/ (FRAC = Fungicide } \\
\text { Resistance Action Committee). Be sure to read a current product label before applying any chemicals, }\end{array}$} \\
\hline
\end{tabular}


Table 8. Selected insecticides approved for use on insect attacking strawberry.

\begin{tabular}{|c|c|c|c|c|c|}
\hline Active Ingredient & Trade Name & Rate & $\begin{array}{l}\text { Re-entry } \\
\text { Interval }\end{array}$ & $\begin{array}{l}\text { Days To } \\
\text { Harvest }\end{array}$ & Pests Controlled \\
\hline Abamectin & AgriMek $0.15 \mathrm{EC}$ & $16 \mathrm{oz} / \mathrm{A}$ & 12 hours & 3 & twospotted spider mites \\
\hline Acequinocyl & Kanenite 15 SC & $21-31 \mathrm{fl} 0 \mathrm{z} . \mathrm{A}$ & 12 hours & 1 & twospotted spider mites \\
\hline \multirow[t]{5}{*}{ Azadirachtin } & Neemix 0.25\% & $0.5-2 \mathrm{gal} / \mathrm{A}$ & 4 hours & 0 & armyworms, caterpillars, loopers \\
\hline & Neemix $4.5 \%$ EC & See label & 12 hours & 0 & armyworms, caterpillars, loopers, thrips \\
\hline & Ecozin 3\% EC & See label & 12 hours & 0 & aphids, beetles, borers, bugs, fruit flies, \\
\hline & Azatin 3\% XL Plus & See label & 4 hours & 0 & $\begin{array}{l}\text { aphids, armyworms, beetles, caterpillars } \\
\text { and loopers, thrips }\end{array}$ \\
\hline & Aza-Direct & See label & 4 hours & 0 & $\begin{array}{l}\text { aphids, armyworms, flies, mites, } \\
\text { thrips, whiteflies }\end{array}$ \\
\hline \multirow{6}{*}{$\begin{array}{l}\text { Bacillus thuringiensis } \\
\text { kurstakie }\end{array}$} & Javelin WG & $0.25-1.5 \mathrm{lbs} / \mathrm{A}$ & 4 hours & 0 & armyworms, cutworms, loopers, \\
\hline & Lepinox WDG & 1-2 lbs/A & 12 hours & 0 & armyworm, loopers \\
\hline & Biobit HP & See label & 4 hours & 0 & armyworms, loopers, \\
\hline & DiPel ES & See label & 4 hours & 0 & armyworm, caterpillar cutworm, looper \\
\hline & DiPel DF & See label & 4 hours & 0 & armyworm, cutworm, \\
\hline & Deliver & See label & 4 hours & 0 & armyworm, cutworm, looper \\
\hline \multirow{5}{*}{$\begin{array}{l}\text { Bacillus thuringiensis } \\
\text { aizawai } \\
\text { Beauveria bassiana }\end{array}$} & Agree WG & $0.5-2 \mathrm{lbs} / \mathrm{A}$ & 4 hours & 0 & armyworms \\
\hline & Xentari & See label & 4 hours & 0 & armyworms, cutworms, loopers, \\
\hline & Botanigard ES & See label & 4 hours & 0 & aphids, thrips \\
\hline & Mycotrol 0 & See label & 4 hours & 0 & aphids, thrips \\
\hline & Naturalis L & $10-15 \mathrm{fl} 0 \mathrm{z} / \mathrm{A}$ & 4 hours & 0 & $\begin{array}{l}\text { ants, aphids, armyworms, loopers, } \\
\text { lygus bug, tarnished plant bug, thrips }\end{array}$ \\
\hline Bifenazate & Acramite 50 WS & $0.75-1 \mathrm{lb} / \mathrm{A}$ & 12 hours & 1 & twospotted spider mite \\
\hline Bifenthrin & Brigade WSB & See label & 12 hours & 0 & $\begin{array}{l}\text { aphids, armyworms, plant bugs, } \\
\text { stink bugs, spider mites }\end{array}$ \\
\hline \multirow[t]{4}{*}{ Carbaryl } & Sevin $4 \mathrm{~F}$ & See label & 12 hours & 7 & $\begin{array}{l}\text { cutworms, omnivorous leaftiers, } \\
\text { tarnished plant bug }\end{array}$ \\
\hline & Sevin 5\% Bait & $40 \mathrm{lbs} / \mathrm{A}$ & 12 hours & 7 & $\begin{array}{l}\text { armyworms, crickets, cutworms, } \\
\text { grasshoppers }\end{array}$ \\
\hline & Sevin $80 \% \mathrm{~S}$ & See label & 12 hours & 7 & $\begin{array}{l}\text { armyworms, cutworms, omnivorous } \\
\text { leaftier, tarnished plant bug }\end{array}$ \\
\hline & Carbaryl $4 \mathrm{~L}$ & See label & 12 hours & 7 & $\begin{array}{l}\text { cutworms, omnivorous } \\
\text { leaftier, tarnished plant bug }\end{array}$ \\
\hline \multirow[t]{4}{*}{ Diazinon } & Diazinon 50W & See label & 24 hours & 5 & aphids, twospotted mites, cyclamen \\
\hline & Diazinon $4 A G$ & See label & 24 hours & 5 & aphids, twospotted mites, cyclamen \\
\hline & Diazinon AG 600 & See label & 24 hours & 5 & aphids, twospotted mites, cyclamen \\
\hline & Diazinon AG 500 & See label & 24 hours & 5 & $\begin{array}{l}\text { aphids, twospotted mites, cyclamen } \\
\text { mites }\end{array}$ \\
\hline \multirow[t]{2}{*}{ Dicofol } & Kelthane 50 WSP & See label & 48 hours & 3 & $\begin{array}{l}\text { cyclamen mites, two spotted spider } \\
\text { mites }\end{array}$ \\
\hline & Dicofol $4 \mathrm{E}$ & See label & 12 hours & 2 & Cyclamen mites, twospotted mites \\
\hline \multirow[t]{2}{*}{ Endosulfan } & Thiodan 50WP & See label & 24 hours & 4 & cyclamen mite, tarnished plant bugs \\
\hline & Phaser 3EC & See label & 24 hours & 4 & cyclamen mite, tarnished plant bugs \\
\hline Etoxazole & Zeal & See label & 12 hours & 1 & $\begin{array}{l}\text { twospotted spider mite, lygus, } \\
\text { spittlebug, tarnished plant bug }\end{array}$ \\
\hline Fenpropathrin & Danitol 2.4 EC & See label & 24 hours & 2 & $\begin{array}{l}\text { lygus, tarnished plant bug, twospotted } \\
\text { spider mites }\end{array}$ \\
\hline Fenbutatin oxide & Vendex 50 WP & $1.5-2 \mathrm{lbs} / \mathrm{A}$ & 48 hours & 1 & twospotted spider mites \\
\hline Hexythiazox & Savey 50 DF & $6 \mathrm{oz} / \mathrm{A}$ & 12 hours & 3 & twospotted spider mite \\
\hline \multirow[t]{5}{*}{ Imidacloprid } & Admire 2 Flowable & $24-32 \mathrm{fl} \mathrm{oz/A}$ & 12 hours & 14 & aphids, whiteflies \\
\hline & Admire Pro & $10.5-14 \mathrm{fl} \mathrm{oz/A}$ & 12 hours & 14 & aphids, whiteflies \\
\hline & Alias $2 \mathrm{~F}$ & $24-32 \mathrm{fl} \mathrm{oz/A}$ & 12 hours & 14 & aphids, whiteflies \\
\hline & Couraze $1.6 \mathrm{~F}$ & $3.8 \mathrm{fl} \mathrm{oz/A}$ & 12 hours & 7 & aphids, whiteflies \\
\hline & Provado 1.6 & $3.75 \mathrm{fl} \mathrm{oz/A}$ & 12 hours & 7 & aphids, whiteflies \\
\hline
\end{tabular}


Table 8. Continued.

\begin{tabular}{|c|c|c|c|c|c|}
\hline Active Ingredient & Trade Name & Rate & $\begin{array}{l}\text { Re-entry } \\
\text { Interval }\end{array}$ & $\begin{array}{l}\text { Days To } \\
\text { Harvest }\end{array}$ & Pests Controlled \\
\hline \multirow[t]{2}{*}{ Malathion } & Malathion 5EC & See label & 12 hours & 3 & $\begin{array}{l}\text { aphids, spider mites, field cricket } \\
\text { lygus bugs, thrips }\end{array}$ \\
\hline & Malathion $8 \mathrm{~F}$ & $1.5-2.0 \mathrm{pt} / \mathrm{A}$ & 12 hours & 3 & $\begin{array}{l}\text { aphids, field crickets, leafhopper, } \\
\text { lygus bugs, spider mites }\end{array}$ \\
\hline \multirow[t]{2}{*}{ Methomyl } & Lannate LV & See label & 48 hours & $\begin{array}{l}\text { 3-Fresh fruit } \\
\text { 10- Processing } \\
\text { fruit }\end{array}$ & $\begin{array}{l}\text { aphids, armyworms, omnivorous } \\
\text { leaftiers, lygus bugs, thrips }\end{array}$ \\
\hline & Lannate SP & See label & 48 hours & $\begin{array}{l}\text { 3-Fresh fruit } \\
10 \text { - Processing } \\
\text { fruit }\end{array}$ & $\begin{array}{l}\text { aphids, armyworms, omnivorous } \\
\text { leaftiers, lygus bugs, thrips }\end{array}$ \\
\hline Methoxyfenozide & Intrepid $2 \mathrm{~F}$ & $6-12 \mathrm{fl} \mathrm{oz}$ & 4 hours & 3 & armyworms, corn earworm, cutworm 1 \\
\hline Naled & DiBrom 8-E & $1 \mathrm{pt} / \mathrm{A}$ & 48 hours & 1 & $\begin{array}{l}\text { leafrollers, spider mites, omnivorous } \\
\text { leaftiers, aphids, thrips, Iygus }\end{array}$ \\
\hline Neem oil & Trilogy & See label & 4 hours & 0 & aphids, mites, whiteflies, thrips ${ }^{1}$ \\
\hline $\begin{array}{l}\text { Potassium salts } \\
\text { of acids (insecticidal) }\end{array}$ & M-Pede & See label & 12 hours & 0 & aphids, leafhoppers, twospotted mites \\
\hline Propargite & Omite CR & $6 \mathrm{lbs} / \mathrm{A}$ & 3 days & $\begin{array}{l}\text { Non-bearing } \\
\text { plants for } 1 \mathrm{yr}\end{array}$ & twospotted spider mite \\
\hline Pyrethrins & PyGanic EC 5.0 & See label & 12 hours & 0 & $\begin{array}{l}\text { aphids, armyworms, beet armyworm, } \\
\text { fruit flies, lygus, tarnished plant bugs, } \\
\text { thrips, whiteflies }\end{array}$ \\
\hline $\begin{array}{l}\text { Pyrethrins \& } \\
\text { Piperonyl Butoxide }\end{array}$ & Evergreen EC 60-4 & $2-16 \mathrm{fl} \mathrm{oz/A}$ & 12 hours & 0 & $\begin{array}{l}\text { aphids, armyworms, beet armyworm, } \\
\text { fruit flies, lygus, tarnished plant bugs, } \\
\text { thrips, whiteflies }\end{array}$ \\
\hline $\begin{array}{l}\text { Pyrethrins \& } \\
\text { Rotenone }\end{array}$ & Pyrellin EC & $1-2 \mathrm{pt} / \mathrm{A}$ & 12 hours & 12 hours & mites, thrips \\
\hline \multirow[t]{2}{*}{ Pyriproxyfen } & Esteem $0.86 \mathrm{EC}$ & $10 \mathrm{fl} \mathrm{oz/A}$ & 12 hours & 2 & $\begin{array}{l}\text { banded wing whitefly, greenhouse } \\
\text { whitefly, silverleaf whitefly }\end{array}$ \\
\hline & Esteem Ant Bait & $1.5-2 \mathrm{lbs} / \mathrm{A}$ & 12 hours & 1 & red imported fire ant \\
\hline (S) methoprene & Extinguish fire ant bait & See label & 4 hours & 0 & fire ants \\
\hline \multirow[t]{3}{*}{ Spinosad } & Entrust & $1.25-1.5 \mathrm{oz} / \mathrm{A}$ & 4 hours & 1 & $\begin{array}{l}\text { armyworms including beet armyworm, } \\
\text { thrips, leaf rollers, omnivorous leaftiers, }\end{array}$ \\
\hline & Spintor 2 SC & $4-6 \mathrm{fl} 0 \mathrm{z} / \mathrm{A}$ & 4 hours & 1 & $\begin{array}{l}\text { armyworms including beet armyworm, } \\
\text { thrips, leaf rollers, omnivorous leaftiers }\end{array}$ \\
\hline & Justice Bait & $2.5-4 \mathrm{lb} / \mathrm{A}$ & 4 hours & 0 & imported fire ants \\
\hline Spiromesifen & Oberon 2SC & $12-16 \mathrm{fl} .0 \mathrm{oz} / \mathrm{A}$ & 12 hours & 3 & Twospotted spider mite, whiteflies \\
\hline Sulfur & Sulfur 6L & $5.33-13 \mathrm{pt} / \mathrm{A}$ & 24 hours & 0 & twospotted spider mite \\
\hline Thiamethoxam & Actara $25 \mathrm{WG}$ & See label & 12 hours & 3 & aphids, whiteflies \\
\hline
\end{tabular}

Table 9. Cost per flat for strawberries at various yield levels in the Plant City area, 2004-2005.

\begin{tabular}{|lcccccc||}
\hline & & \multicolumn{5}{c||}{ Yield (flats/acre) } \\
\cline { 3 - 7 } & Cost per acre & $\mathbf{2 , 4 0 0}$ & $\mathbf{2 , 5 0 0}$ & $\mathbf{2 , 6 0 0}$ & $\mathbf{2 , 7 0 0}$ & $\mathbf{2 , 8 0 0}$ \\
Variable Costs & $\$ 7,612.36$ & $\$ 3.17$ & $\$ 3.04$ & $\$ 2.93$ & $\$ 2.82$ & $\$ 2.72$ \\
Fixed Costs & $\$ 3,858.18$ & $\$ 1.61$ & $\$ 1.54$ & $\$ 1.48$ & $\$ 1.43$ & $\$ 1.338$ \\
Harvest Cost/unit & & $\$ 5.60$ & $\$ 5.60$ & $\$ 5.60$ & $\$ 5.60$ & $\$ 5.60$ \\
Total Cost/unit & $\$ 10.38$ & $\$ 10.19$ & $\$ 10.01$ & $\$ 9.85$ & $\$ 9.70$ \\
\hline
\end{tabular}

